\section{ORIGINAL RESEARCH}

\author{
J.J. Kim
}

W.P. Dillon

C.M. Glastonbury

J.M. Provenzale

M. Wintermark

\title{
Sixty-Four-Section Multidetector CT Angiography of Carotid Arteries: A Systematic Analysis of Image Quality and Artifacts
}

\begin{abstract}
BACKGROUND AND PURPOSE: Sixty-four-section CT scanners have recently been introduced for vascular imaging. Before such scanners reach widespread use, scanning protocol should be optimized and image quality assessed. The goals of this study were to systematically measure image quality and determine the prevalence of various types of artifacts produced by a 64-section scanner.
\end{abstract}

\begin{abstract}
MATERIALS AND METHODS: We retrospectively reviewed CT angiography (CTA) scans obtained on a 64-section CT scanner in 100 consecutive patients presenting to the emergency department during a 2-month period with a suspected acute cerebrovascular event. We evaluated scan quality by using 2 different methods: First, we quantitatively assessed arterial opacification by measuring attenuation values in 9 arterial segments from the aortic arch to the distal cervical internal carotid artery, by using a threshold of $150 \mathrm{HU}$ as an indicator of good opacification. Second, we assessed image contrast between arteries and veins by measuring attenuation within venous segments and recording the number of artery-vein-segment pairs in which the attenuation difference was $\leq 50 \mathrm{HU}$. In addition, we recorded the prevalence of the following artifacts: metallic hardware streak, contrast material streak from slow-flowing contrast material in adjacent large veins, streak artifacts from shoulders, contrast material reflux into veins of the neck, motion artifacts, and artifacts causing misrepresentation of flow dynamics simulating arterial dissection or occlusion. These results were compared with those of a historical control group of 113 patients from our institution who were imaged with the same technical parameters on a 16-section CT scanner.
\end{abstract}

RESULTS: The quantitative assessment of arterial opacification showed that 854 of 885 analyzed arterial segments $(96.5 \%$ ) had good opacification (ie, attenuation values $>150 \mathrm{HU}$ ). Image contrast between artery and vein segments was also good, with 714 of 763 analyzable segment pairs (85.6\%) having $>50 \mathrm{HU}$ difference. Artifacts obscuring arterial evaluation included streak from contrast material in the subclavian/brachiocephalic vein (32\% of patients), attenuation of the x-ray beam between the shoulders $(28 \%)$, beam-hardening from metallic hardware $(26 \%)$, and contrast material reflux into neck veins $(16 \%)$. The most clinically relevant artifacts were flow artifacts, mimicking dissection or vascular occlusion; they were seen in $14 \%$ of patients and likely are related to the rapid data acquisition for CTA on 64-section scanners (compared with the circulation of contrast material in the cervical arteries). None of the patients in our historical control group who underwent 16-section CT had flow artifacts on their CTA studies; the incidence of the other types of artifacts in this group was similar to that in patients imaged with 64-section CT.

CONCLUSIONS: The 64-section CTA imaging protocol for carotid arteries yields high-quality studies in $>95 \%$ of cases.

$I^{\mathrm{n}}$ $\mathrm{n}$ the acute emergency department setting, there is increasing reliance on CT angiography (CTA) for triage and evaluation of patients with suspected cerebrovascular disease. In patients with symptoms of stroke or transient ischemic attack, CTA can swiftly and accurately assess the patency of the intracranial circulation, evaluate for carotid atherosclerosis as a potential embolic source, and provide information about vessel occlusion that is essential before treatment with thrombolysis or even embolectomy is considered. ${ }^{1-3}$ The wide availability, relative safety in acute urgent settings, and speed of CTA, which is accessible anytime day or night, make it preferable to

Received April 21, 2009; accepted after revision June 4.

From the Department of Radiology (J.J.K., W.P.D., C.M.G., M.W.), Neuroradiology Section, University of California, San Francisco, San Francisco, California; Department of Radiology (J.M.P.), Duke University Medical Center, Durham, North Carolina; and Departments of Radiology, Oncology and Biomedical Engineering, (J.M.P.), Emory University School of Medicine, Atlanta, Georgia.

Please address correspondence to Max Wintermark, MD, University of California, San Francisco, Department of Radiology, Neuroradiology Section, 505 Parnassus Ave, Box 0628, San Francisco, CA 94143-0628; e-mail: Max.Wintermark@radiology.ucsf.edu

DOI 10.3174/ajnr.A1768
MR imaging/MR angiography as a first-line diagnostic tool for patients in the emergency department suspected of having acute cerebrovascular events.

Furthermore, CTA image quality has benefited during the past decade from the steady stream of improvements of multidetector CT scanners introduced by commercial vendors, from single-detector-row to 4-, 8- and 16-section scanners. Sixty-four-section scanners were first introduced in 2004 and have become increasingly popular in clinical use, reflecting current state-of-the-art stroke CTA imaging. Sixty-foursection CTA from the aortic arch through the intracranial vessels can be performed in 3-4 seconds at submillimeter isotropic resolution with superb-quality multiplanar reformats, but image quality benefits from a dedicated acquisition protocol to address the new challenges of fast CT scanning.

In particular, correct timing of the contrast material bolus to ensure good arterial opacification and high CTA image quality becomes even more important with 64-section CT scanners, in which the rapid CT acquisition can outpace the flow of contrast-opacified blood. Given the increased adop- 
tion of 64-section CTA, the purpose of this article is to describe our recommended carotid CTA imaging protocol for 64section scanners, evaluate our initial results, and discuss potential pitfalls and artifacts, some of which may be unique to 64-section CTA.

\section{Materials and Methods}

\section{Overview of Stroke Protocol}

The 64-section stroke CT protocol used at our institution as the standard of care for each patient suspected of having stroke (64-section LightSpeed Volume CT; GE Healthcare, Milwaukee, Wisconsin) consists of 4 series obtained in the following order: 1) unenhanced head CT, 2) perfusion CT performed at 2 levels in the brain, 3) CTA from the base of the heart through the cerebral vertex, and 4) contrastenhanced head CT.

Following the unenhanced head CT, perfusion CT is performed at 2 levels, that of the third ventricle and again more cephalad at a point above the lateral ventricles. At each level, $40-\mathrm{mL}$ iodinated contrast material (iohexol, Omnipaque $350 \mathrm{mg} \mathrm{I} / \mathrm{mL}$; GE Healthcare) followed by $25-\mathrm{mL}$ saline is administered, and a series of dynamic lowdose scans $(80 \mathrm{kV}, 100 \mathrm{~mA})$ is obtained during injection. Contrast material is injected at $5 \mathrm{~mL} / \mathrm{s}$, the same rate used for CTA.

Dynamic scanning during contrast material injection not only generates data for perfusion CT analysis but also serves as a timing bolus to calculate scanning delay between intravenous injection and contrast arrival in the neck and brain for CTA. At the first perfusion CT level, arterial enhancement is plotted against time by using a region of interest placed in a branch of the anterior cerebral artery by the technologist. The time-to-peak enhancement of the arterial timeenhancement curve is determined for each patient (usually around 8 seconds) and added to the standard delay between time from contrast material injection to initiation of perfusion CT scanning (7 seconds), yielding a total scanning delay of 15 seconds following initiation of contrast injection in this example. One second is then empirically deducted from the scanning delay (14 seconds in this example) because opacification will occur slightly earlier in the cervical carotid arteries compared with the region of interest placed over a branch of the intracranial circulation evaluated by the timing-bolus technique. Patients with impaired cardiac function, atrial fibrillation, or significant vascular stenoses will usually have longer time-to-peak arterial enhancement and thus longer scanning delays than otherwise healthy patients.

Once the appropriate scanning delay has been determined, CTA is performed with $70-\mathrm{mL}$ iodinated contrast material (iohexol, Omnipaque $350 \mathrm{mg} \mathrm{I} / \mathrm{mL}$ ) followed by a $25-\mathrm{mL}$ saline bolus chaser at $5-\mathrm{mL} / \mathrm{s}$ injection rate. Scanning parameters for CTA are as follows: number of detectors, 64; gantry rotation time, 0.4 seconds; caudocranial acquisition; collimation, $0.625 \mathrm{~mm}$; pitch, 0.984:1; $120 \mathrm{kV}$; 200-400 mA; dose modulation with noise index of 12 . Sections are reconstructed at $1.25-\mathrm{mm}$ thickness with a $1-\mathrm{mm}$ reconstruction interval for evaluation of the vasculature, while the $0.625-\mathrm{mm}$ raw data images are used to create maximum-intensity-projection (MIP) images. Sections are also reconstructed at $2.5-\mathrm{mm}$ thickness with a $2-\mathrm{mm}$ reconstruction interval for evaluation of soft tissues. Finally, contrast-enhanced head CT is performed approximately 1 minute after CTA (without the need for additional contrast material injection because contrast material has already been administered for perfusion CT and CTA). This provides additional information regarding incidental lesions, subacute infarctions, contrast extravasation in cases of intracranial hematoma or aneurysm rupture, and a delayed view of vessels that appear occluded on initial CTA. The total amount of contrast material is $150 \mathrm{~mL}(2 \times 40 \mathrm{~mL}$ for CT perfusion and $70 \mathrm{~mL}$ for CTA). The total duration of the examination from start to finish is approximately 8 minutes.

For contrast material infusion, an 18-gauge intravenous catheter is typically used. The right arm is preferentially selected whenever possible to avoid streak artifacts in the mediastinum from contrast material retained in the left subclavian vein with a left-arm injection. These artifacts are frequently encountered in elderly patients when the brachiocephalic vein is compressed between the sternum and an ectatic aorta arch. The antecubital fossa is preferred for intravenous access over arm-vein access, which in turn is preferable to hand-vein access.

Postprocessing is performed by the technologist at the scanner or in the 3D laboratory. MIP images are obtained of the level of the circle of Willis in axial, coronal, and sagittal planes; of the carotid bifurcations bilaterally in sagittal oblique planes; and of the origins of the great vessels and extracranial carotid arteries in the coronal plane. Additional computer-aided analysis of the degree of carotid artery stenosis is undertaken by using the North American Symptomatic Carotid Endarterectomy Trial criteria, in accordance with Medicare reimbursement. ${ }^{4}$

\section{Patient Population}

This was a retrospective study performed under the auspices of the institutional review board at our hospital. Patients were included in the study under a waiver of informed consent. We reviewed stroke CT studies of 100 consecutive patients who presented to the emergency department and underwent the 64-section CT stroke protocol described above between August 11, 2007, and October 4, 2007 (48 men, 52 women; mean age, 65.5 years; age range, $23-98$ years). Indications for stroke protocol included suspected stroke in 82 patients, suspected transient ischemic attack in 11, and suspected carotid artery dissection in 7. All CTA studies and contrast-enhanced CT scans were reviewed; unenhanced and perfusion CT scans were not evaluated for this study.

\section{Measurement of Opacification of Artery and Vein Segments}

For each CTA study, the side of contrast material injection (right or left) was recorded. Most CTA examinations $(n=63)$ were performed with contrast material injected into the right arm. The quality of arterial opacification was assessed by 1 radiologist (J.J.K.) with 6 years of experience in evaluating stroke CTA, who measured attenuation in Hounsfield units at 9 locations: ascending aorta (at the level of the main pulmonary artery bifurcation), right and left proximal common carotid arteries (CCAs, at the level of the inferior aspect of the cricoid cartilage), right and left distal CCAs (at the level of the hyoid bone), right and left proximal internal carotid arteries (ICAs, just beyond the carotid bifurcation), and right and left distal cervical ICAs (at the level of the anterior arch of $\mathrm{C} 1$ ).

Attenuation values of the veins at 9 corresponding locations were also noted; measurements were made in the superior vena cava and internal jugular vein (IJV) on each side adjacent to the arterial measurement. To obtain the attenuation value, a circular region of interest (of the greatest diameter that would fit in the vessel lumen) was drawn on axial images in the center of the lumen. The typical size for such regions of interest was $20 \mathrm{~mm}^{2}$. The mean, SD, and maximum and minimum attenuation values were recorded for each arterial and 
venous location. Based on a review of the literature, we deemed arterial attenuation values $\geq 150 \mathrm{HU}$ be optimal for $\mathrm{CTA}^{5,6}$ and counted the number of arterial segments with attenuation values $>150 \mathrm{HU}$. To evaluate the degree of image contrast between arteries and veins, we calculated the difference in attenuation between artery and vein for each considered level. On the basis of a similar analysis by Rubin et $\mathrm{al}^{6}$ of attenuation differences between arteries and veins and the requirements of intensity-based software for $3 \mathrm{D}$ rendering purposes, we considered that a threshold difference of $\geq 50$ HU between arterial and venous attenuation was optimal, and the number of segments with $>50 \mathrm{HU}$ attenuation difference between arteries and corresponding veins was recorded.

\section{Recording of Artifacts}

A single observer (J.J.K.) recorded artifacts that caused partial or significant obscuration of adjacent arteries and assigned them to 1 of the following categories: 1) metallic hardware streak (eg, in the upper chest from cardiac pacer leads or in the neck from dental hardware); 2) streak artifacts of venous origin (ie, slow-flowing contrast material in the subclavian vein, brachiocephalic vein, and/or superior vena cava obscuring the origins of great vessels); 3) streak artifacts between the shoulders from photon starvation; 4) contrast material reflux into small neck veins (ie, contrast in the vertebral venous plexus obscuring the adjacent vertebral artery), 5) motion, and 6) flow artifacts or misrepresentation of flow dynamics (eg, pseudodissection or pseudoocclusion). Also, the degree of contrast material reflux into the IJV was determined by measuring the caudocranial length of reflux into the IJV from the confluence of the IJV and the brachiocephalic vein as depicted on a coronal MIP image. The side of contrast material injection was compared with the extent of contrast material reflux into the IJV by using the Wilcoxon rank sum test for nonparametric data.

\section{Historical Control Group}

We compared the quality of arterial opacification and the incidence of artifacts on 64-section CTA examinations with those on 16-section studies by using a historic control group of patients from our institution who had been previously studied and published. ${ }^{7}$ This control group consisted of 113 consecutive patients over a 2 -year period who were suspected of having acute hemispheric stroke and underwent stroke protocol imaging on a 16-section scanner, consisting of unenhanced CT, perfusion CT, CTA, and delayed contrast-enhanced CT. Scanning parameters, including contrast dose and determination of scanning delay by test bolus technique (by using perfusion CT), were the same in this group as for the 64-section CTA. The quality of arterial opacification and incidence of artifacts between these patient groups were compared by using the Fisher exact test.

\section{Results}

\section{Quantitative Assessment of Arterial Opacification on Carotid CTA}

Of 900 possible arterial segments (ie, 9 locations in 100 patients), 885 segments (98.3\%) were included in the analysis; 15 segments were excluded because of arterial occlusion as determined by qualitative assessment of the CTA. In general, the intensity of arterial opacification on CTA was high throughout, with mean arterial attenuation averaged across all segments equal to $309.9 \pm 92.6 \mathrm{HU}$. Figure 1 charts the mean attenuation \pm SD for each arterial location. The maximum arterial attenuation was $600 \mathrm{HU}$, and the minimum attenua-

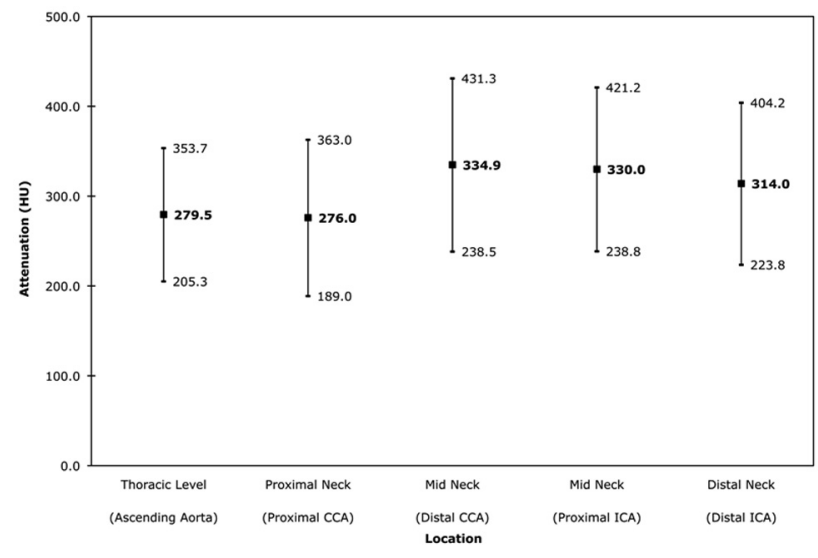

Fig 1. Intraluminal attenuation by arterial location. The mean arterial attenuation is shown in bold (center value). Values at 1 SD above and below the mean are also noted. CCA indicates common carotid artery; ICA, internal carotid artery.

tion was $83 \mathrm{HU} ; 854$ of 885 segments $(96.5 \%)$ had attenuation $>150 \mathrm{HU}$. The 31 measurements with attenuation $<150 \mathrm{HU}$ were obtained in the ascending aorta $(n=3)$, proximal CCA $(n=14)$, distal CCA $(n=2)$, proximal ICA $(n=5)$, and distal ICA $(n=7)$.

The quality of arterial opacification achieved on 64-section CTA was not significantly different from that achieved in our 16-section CTA historical control group. In this control group of 113 patients and 1012 total arterial segments (9 segments per patient as in the current study with exclusion of 5 occluded segments), 967 of 1012 segments (95.5\%) had attenuation $>150 \mathrm{HU}(P=.348$ for Fisher exact test $)$.

The side of contrast material injection had no influence on mean arterial attenuation $(P=.838$ for the 2 -tailed $t$ test $)$.

\section{Quantitative Assessment of Image Contrast between Arteries and Veins}

Of 900 possible venous segments, 778 segments (86.4\%) were considered for analysis. Venous segments containing noncirculated undiluted contrast material (all 100 measurements in the superior vena cava and 22 measurements in the IJV with contrast material reflux) were excluded. Mean venous opacification averaged across all segments was $133.8 \pm 60.6 \mathrm{HU}$, and the mean attenuation difference between artery and vein, averaged across all locations, was $180.8 \pm 88.2 \mathrm{HU}$. By location, attenuation difference was $183.6 \pm 82.2 \mathrm{HU}$ in the proximal CCA, 203.1 \pm 87.9 HU in the distal CCA, $183.6 \pm 85.9$ $\mathrm{HU}$ in the proximal ICA, and $153.3 \pm 89.7 \mathrm{HU}$ in the distal ICA. The number of artery- and vein-segment pairs with $>50-\mathrm{HU}$ attenuation difference was 714 of 763 segments $(85.6 \%)$. (Note that the 15 arterial segments with occlusion were not included in this analysis.) Twenty-three of 49 segments showing $<50 \mathrm{HU}$ attenuation difference were in the distal neck (distal ICA/IJV at C1), which generally showed the highest levels of venous opacification.

\section{Artifacts}

The prevalence of different types of artifacts encountered on 64-section CTA compared with 16-section CTA is listed in the Table and illustrated in Fig 2. Most of the artifacts we encountered caused obscuration of vessels.

Contrast material reflux into the IJV was observed in a total 


\begin{tabular}{|c|c|c|c|c|}
\hline \multicolumn{5}{|c|}{ Artifacts and problems limiting artery evaluation } \\
\hline Type of Artifacts & Description & $\begin{array}{l}\text { No. of Cases } \\
\text { (incidence) on } \\
\text { 64-Section } \\
\text { CTA }\end{array}$ & $\begin{array}{l}\text { No. of Cases } \\
\text { (incidence) on } \\
\text { 16-Section } \\
\text { CTA }\end{array}$ & $\begin{array}{c}P \text { Value } \\
\text { (Fisher exact test) }\end{array}$ \\
\hline Patient motion artifacts & $\begin{array}{l}\text { Gross motion during scan } \\
\text { acquisition }\end{array}$ & $0(0 \%)$ & $3(2.7 \%)$ & .247 \\
\hline Streak artifacts of venous origin & $\begin{array}{l}\text { Dense undiluted contrast in } \\
\text { SCV, BCV; SVC obscures } \\
\text { great vessel origins }\end{array}$ & $32(32 \%)$ & $29(25.7 \%)$ & .363 \\
\hline Shoulder streak artifacts & $\begin{array}{l}\text { Photon starvation between } \\
\text { shoulders obscures CCA, } \\
\text { VA, IJV in lower neck }\end{array}$ & $28(28 \%)$ & $33(29.2 \%)$ & .880 \\
\hline Metallic streak artifacts & $\begin{array}{l}\text { Dental hardware, cardiac } \\
\text { defibrillator leads, spinal } \\
\text { fusion hardware obscure } \\
\text { adjacent arteries }\end{array}$ & $26(26 \%)$ & $32(28.3 \%)$ & .759 \\
\hline Contrast material reflux & $\begin{array}{l}\text { Contrast reflux into veins } \\
\text { of neck obscures } \\
\text { adjacent VA }\end{array}$ & $16(16 \%)$ & $17(15 \%)$ & .852 \\
\hline $\begin{array}{l}\text { Flow artifacts: } \\
\text { Arterial }\end{array}$ & $\begin{array}{l}\text { Altered flow dynamics (eg, } \\
\text { slow flow) and rapid }\end{array}$ & $14^{\mathrm{a}}(14 \%)$ & $0(0 \%)$ & $<.001$ \\
\hline Pseudo-dissection & scanning time create & 5 & & \\
\hline Pseudo-occlusion & appearance of dissection & 6 & & \\
\hline Differential attenuation & flap or nonopacification & 1 & & \\
\hline Venous & of patent vessel & & & \\
\hline Pseudo-thrombosis & & 3 & & \\
\hline
\end{tabular}

Note:-CCA indicates common carotid artery; BCV, brachiocephalic vein; IJV, internal jugular vein; SCV, subclavian vein; SVC, superior vena cava; VA, vertebral artery; CTA, CT angiography ${ }^{a}$ One patient had both pseudodissection and pseudo-occlusion.
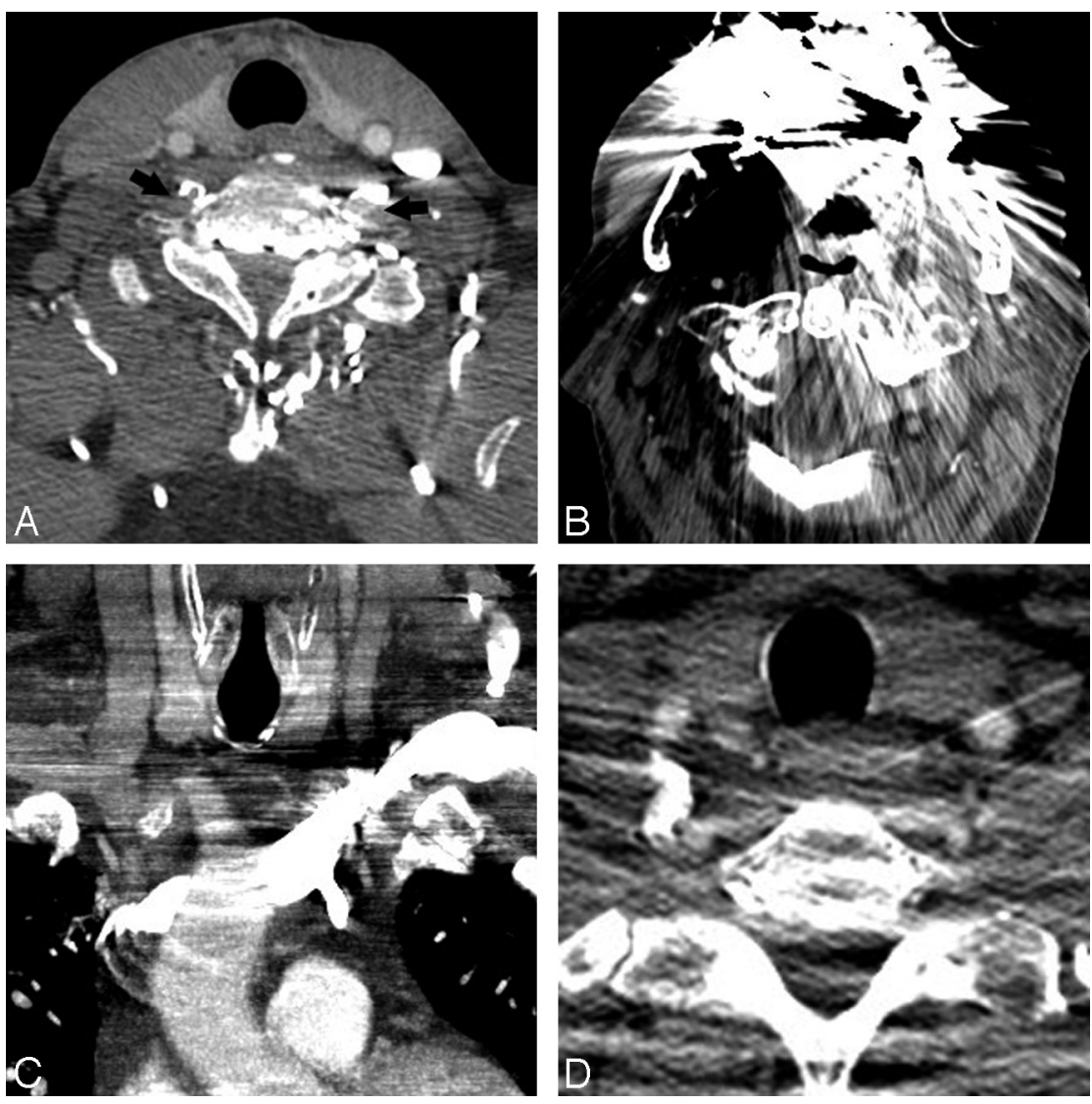

Fig 2. Artifacts interfering with CTA interpretation. $A$, Contrast reflux into small neck veins obscures evaluation of the vertebral arteries (black arrows). $B$, Significant streak artifacts from dental hardware limit evaluation of the carotid and vertebral arteries. $C$, Left-sided injection results in attenuated contrast opacification of the left brachiocephalic vein, which obscures the origin of the great vessels. $D$, Photon starvation between the shoulders results in streaks of low attenuation that make evaluation of the vertebral arteries difficult. 

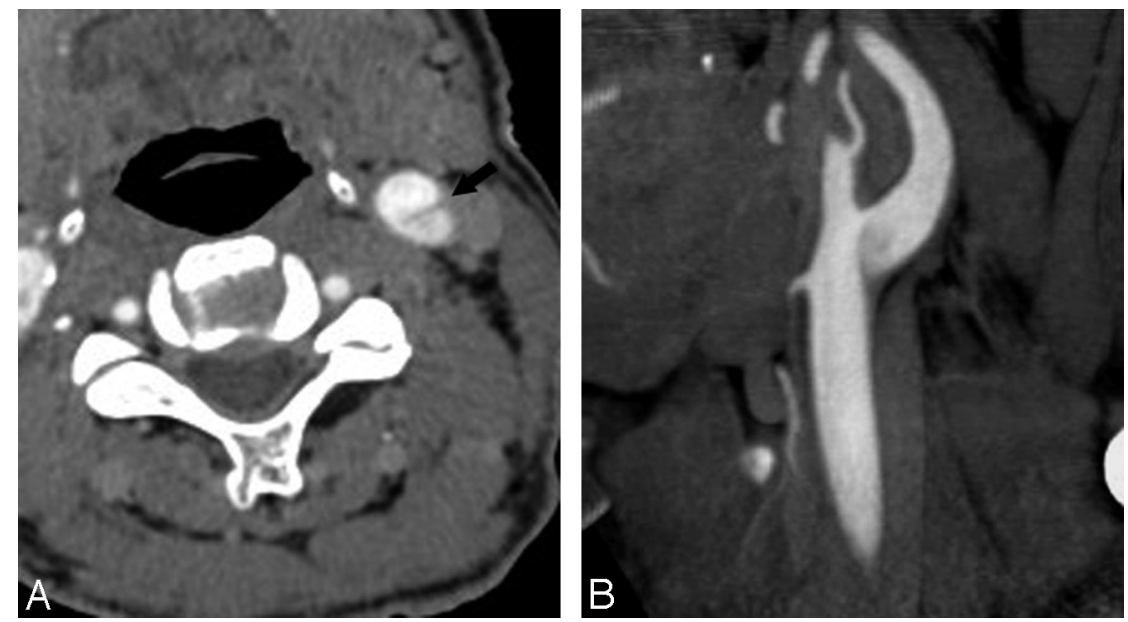

Fig 3. A 68-year-old man with pseudodissection of the left carotid bifurcation. $A$ and $B$, Axial source $(A)$ and sagittal reformatted maximum-intensity-projection (MIP) image from CTA ( $B$ ) demonstrate faint linear flap extending vertically from the left carotid bifurcation and the appearance of 2 vascular compartments with different degrees of opacification
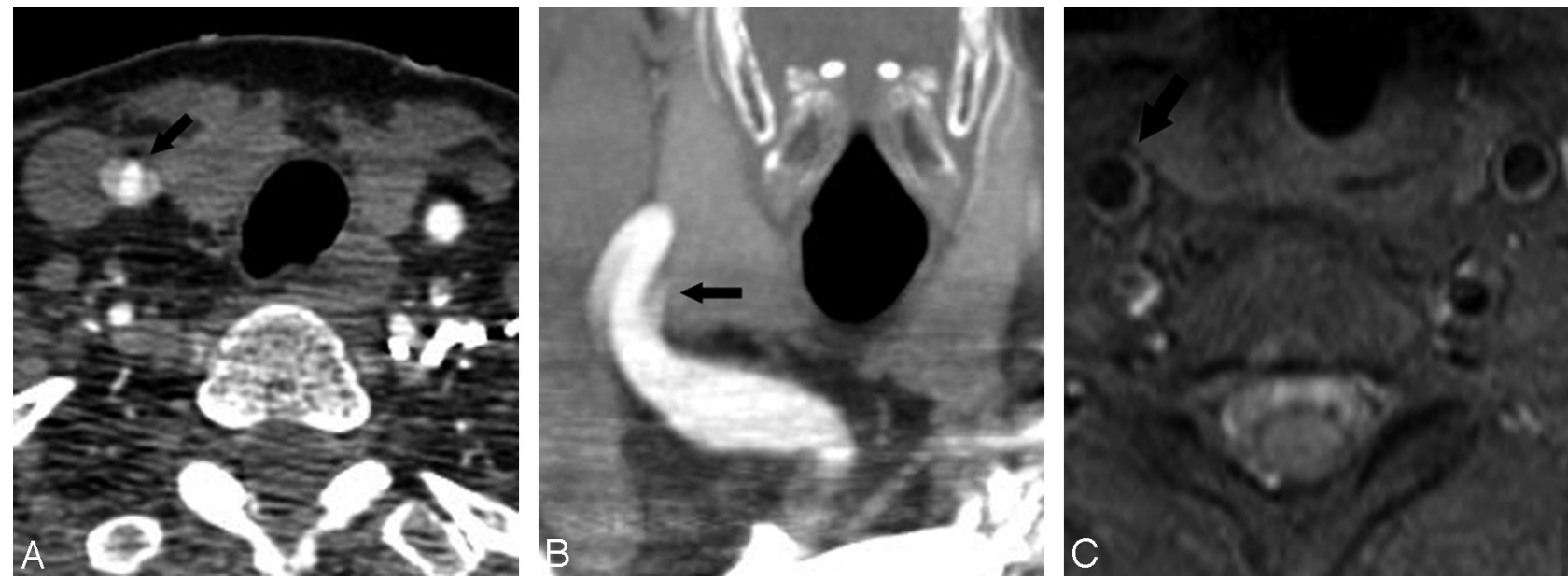

Fig 4. A 61-year-old woman with pseudodissection of the proximal right CCA. $A$ and $B$, Axial source $(A)$ and coronal reformatted MIP image from CTA (B) shows a trilaminar appearance of the proximal right CCA (arrow), disconcerting for dissection. $C$, Axial T1-weighted image using a fat saturation technique through the cervical carotid artery (arrow), obtained immediately after the CT study, shows no evidence of dissection. The finding on CTA is considered to represent pulsation artifacts.

of 50 patients ( 30 of 63 patients with right-sided injections and 20 of 37 patients with left-sided injections). The median length of reflux measured $1.1 \mathrm{~cm}(0.80-2.80 \mathrm{~cm}$ for the 25 th-75th percentile, respectively). However, there was no significant difference between right- and left-sided injections in terms of median length of reflux in the IJV $(P=.745$, Wilcoxon rank sum).

There was no significant difference between 64 - and 16section CTA in the incidence of artifacts except for those related to flow dynamics. These artifacts were seen in 14\% of 64-section CTA studies but were not present in any of the 16-section CTA studies in our historical control group. Unlike the artifacts that produced obscuration of vessels, artifacts related to flow dynamics caused difficulties with assessing the type of flow within vessels. Two types of artifacts were produced by flow in the current study (ie, a pseudodissection appearance and a pseudo-occlusion appearance). These will be discussed in turn.

One type of artifact produced by flow dynamics simulated the appearance of arterial dissection. A linear hypoattenuated stripe was noted within the arterial lumen in 5 cases, which could have been mistaken for a dissection flap if not evaluated carefully. All cases involved the carotid artery, 3 of which occurred in the ICA immediately distal to the carotid bifurcation (Fig 3). These were presumed to be artifacts on the basis of the faintness of the low-attenuation flap and the typical location for flow reversal or separation as depicted on Doppler sonograms and conventional angiograms (further discussion below). None of these 3 patients had follow-up imaging. However, 2 patients had similar apparent dissection flaps in other locations ( 1 in the proximal CCA [Fig 4] and the other in the distal CCA extending into both internal and external carotid arteries). In these cases, the appearance was sufficiently disturbing that subsequent imaging was recommended; neither patient was found to have dissection.

The second type of flow-related artifacts was one of arterial pseudo-occlusion. In 6 patients, a portion of vessel was not opacified and thus was apparently occluded on CTA. However, these segments were subsequently presumed to be patent on the basis of opacification on delayed contrast-enhanced CT. These findings suggest that flow was present but sluggish; thus, it is likely that the very rapid CTA acquisition outpaced the relatively slow flow of contrast-opacified blood in these individuals. While all 6 patients were found to have segments 

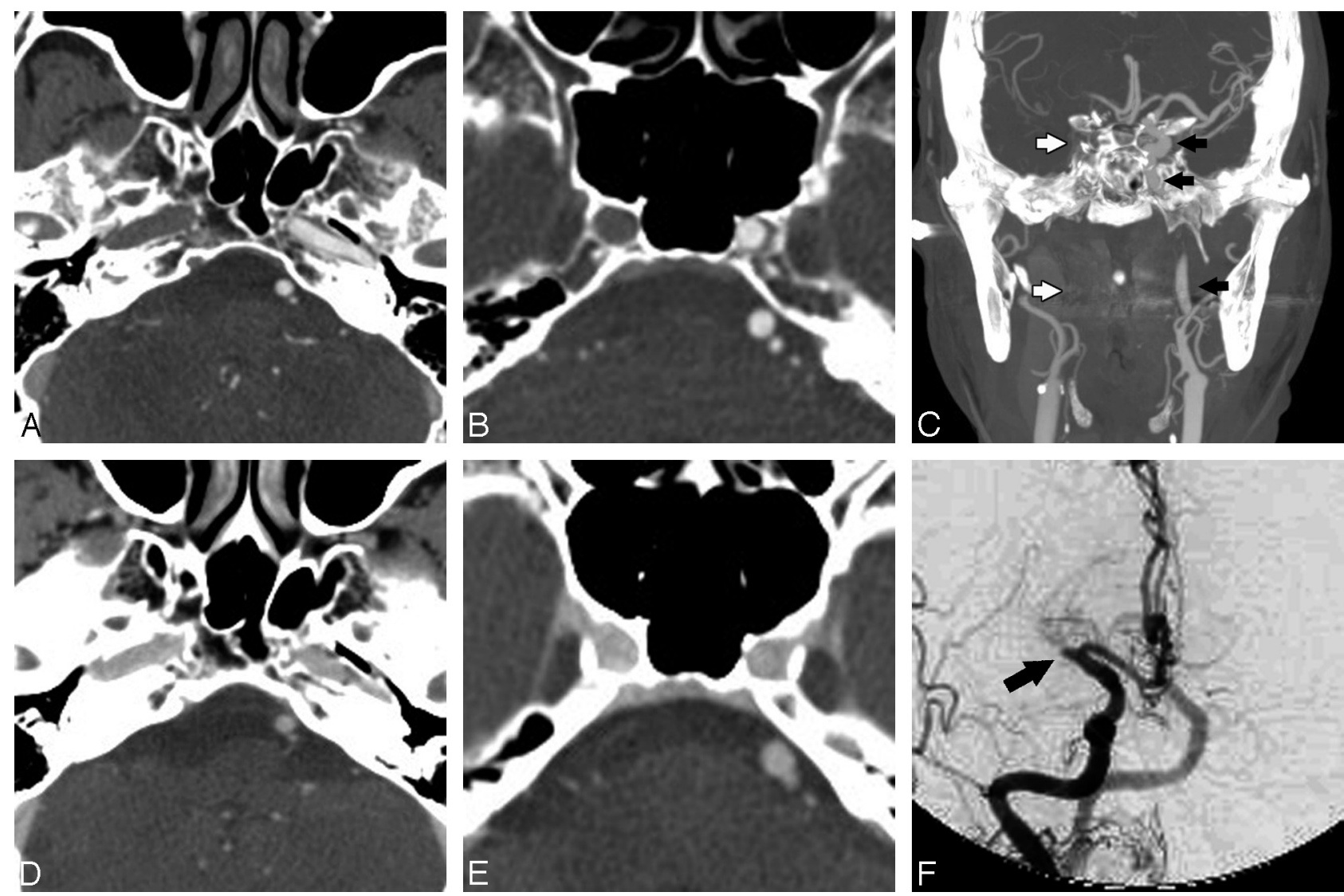

Fig 5. A 79-year-old woman with left hemiparesis. $A-C$, CTA shows nonopacification and apparent occlusion of the entire right ICA, from its origin to the carotid terminus. Compare the normally opacified left ICA (black arrows) with the nonopacified right side (white arrows in C denote the expected course of the right ICA). D and E, Axial contrast-enhanced images, however, show opacification and confirm patency of the right ICA at comparable levels. F, Conventional angiogram obtained 4 hours later (anteroposterior [AP] view right carotid injection) confirms patency of the entire intracranial ICA but reveals an abrupt cutoff of the proximal M1 segment (arrow), consistent with occlusion. Nonopacification on CTA is attributed to the sluggish flow resulting from the $\mathrm{M} 1$ occlusion on the right side.

of vessel that were occluded, CTA overestimated the extent of occlusion compared with contrast-enhanced CT (Fig 5). Five patients had CTA images showing occlusion of variable lengths in the ICA and middle cerebral artery (MCA); on delayed contrast-enhanced CT, however, some portions of the intracranial and distal cervical ICA that appeared occluded on CTA showed contrast material filling, with the occlusion being limited to the ICA terminus or the M1 segment of the MCA. The sixth patient had a vertebral artery occlusion, the extent of which was overestimated by CTA compared with contrast-enhanced CT. Follow-up imaging (1 MR angiogram and 2 conventional angiograms) was available in 3 patients to confirm vessel patency; the other 3 patients in whom follow-up imaging was not available were presumed to have pseudo-occlusion.

To determine the reason for slow arterial flow causing confirmed or presumed pseudo-occlusion on CTA, we reviewed the entire course of affected arteries to determine if proximal stenoses caused impedance to flow. Such proximal stenoses were, in fact, identified in 3 of the 6 patients. Hemodynamically significant proximal stenoses were seen due to atherosclerotic narrowing in 2 patients and a type A aortic dissection, in 1 . However, in the remaining 3 patients, no hemodynamically significant proximal stenosis was appreciated but distal occlusions were noted (MCA occlusion in 1 patient and carotid terminus occlusions in 2 patients).
Besides causing artifactual occlusion on CTA, distal thrombosis may also create the appearance of differential attenuation between the right and left carotid arteries through altered flow dynamics. One patient in our study had a right MCA territory stroke due to thromboembolism in the M1 segment of the MCA. Rather than nonopacification and pseudo-occlusion on CTA as in the cases above, the right intracranial ICA showed diffusely decreased attenuation compared with the left side on CTA (Fig 6). No proximal stenosis was identified to account for the slower flow and lower attenuation of the right ICA.

Finally, venous flow artifacts were noted in 3 patients. Similar to the cases of arterial pseudo-occlusion and overestimation of occlusion by CTA compared with contrast-enhanced CT, 1 patient with a right-sided central line showed apparent thrombosis of the entire ipsilateral IJV on CTA. The visualized IJV at the skull base on contrast-enhanced CT, however, had delayed opacification consistent with at least partial patency and slow blood flow (Fig 7). The other 2 patients had pseudothrombosis of the IJV at the insertion of a smaller vein as it emptied a column of nonopacified blood into the IJV (Fig 8).

\section{Discussion}

The major findings in this study are as follows: First, we showed that, with the CTA protocol used in this study, very good arterial opacification and substantial differences in de- 

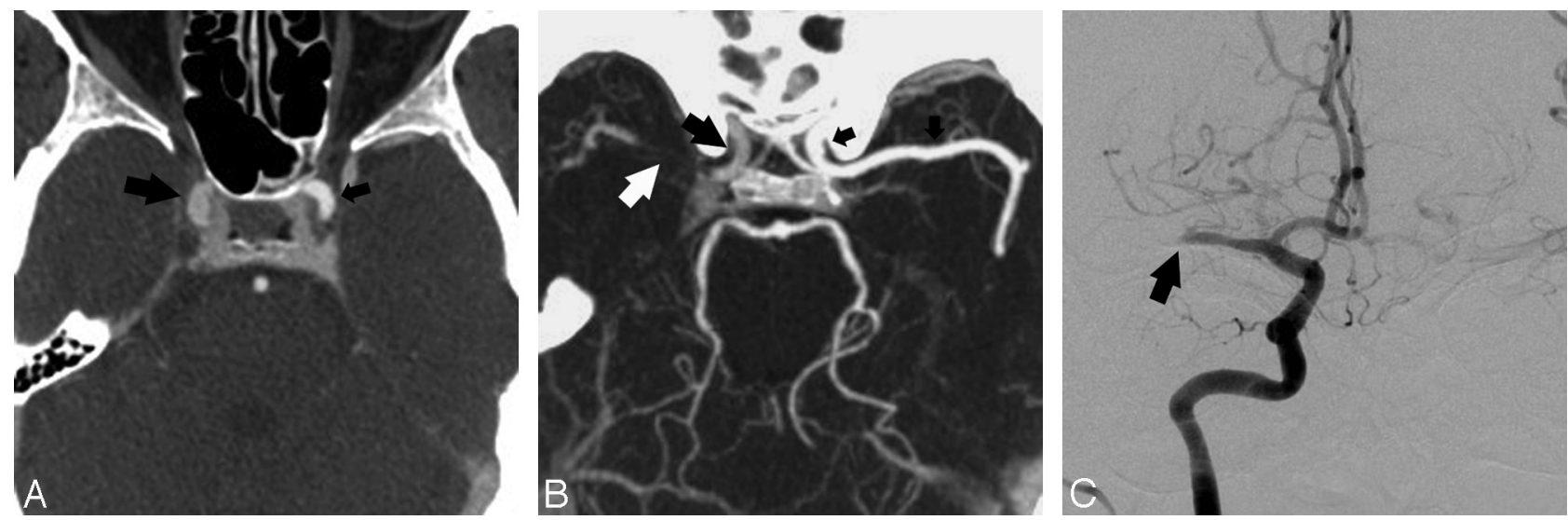

Fig 6. A 71-year-old woman with left-sided hemiparesis. A, Axial source CTA image shows opacification but decreased attenuation of the right supraclinoid ICA (large arrow) compared with the normal left side (small arrow). B. Axial reformatted MIP image again demonstrates overall decreased attenuation of the right supraclinoid ICA (large black arrow) compared with the normal left ICA and middle cerebral artery (MCA, small black arrows). A large filling defect is noted in the right MCA (white arrow). C, Angiogram, AP-view right carotid injection, shows an abrupt cutoff (black arrow) in the right MCA consistent with occlusion. No hemodynamically significant stenoses were observed at the origin or along the course of the right carotid artery to account for impaired blood flow and decreased arterial attenuation, which was instead attributed to the presence of a distal thrombus.
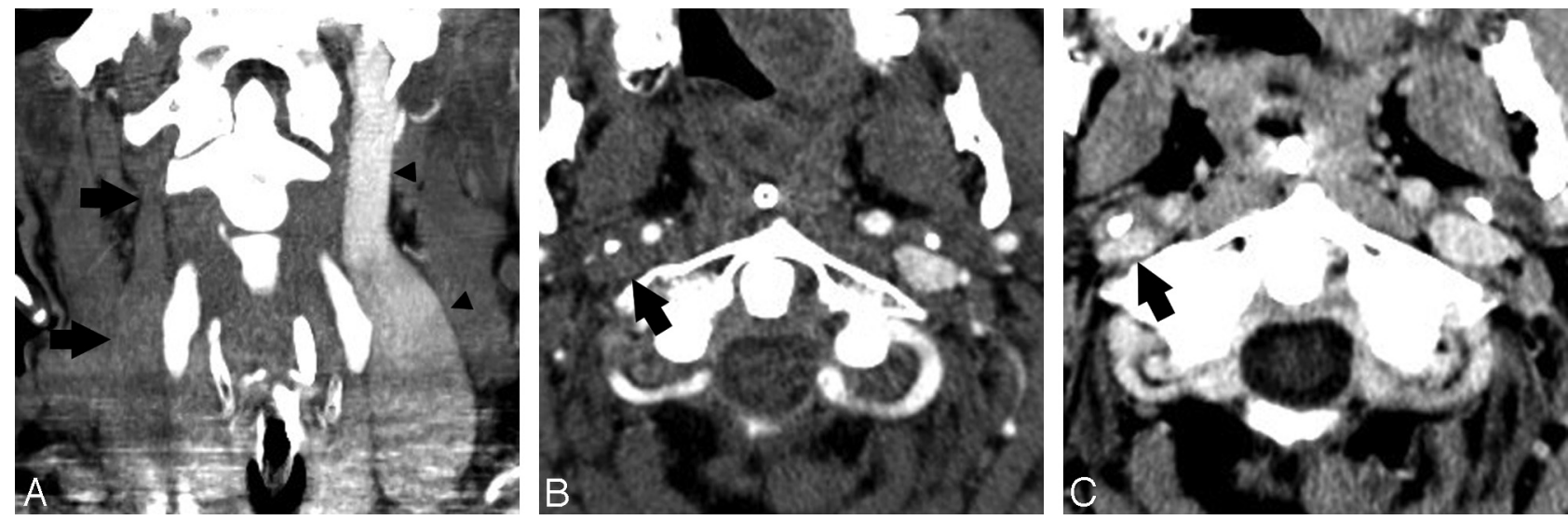

Fig 7. A 41-year-old man with apparent thrombosis of the entire right internal jugular vein (IJV). $A$, Coronal reformatted MIP image from CTA shows nonopacification of the entire visualized right internal jugular vein (IJV, arrows) compared with the opacified left IJV (arrowheads). B, Axial source CTA image again shows no contrast in the right IJV (arrow). C, Contrast-enhanced CT at the same level as $B$ shows delayed contrast filling of the IJV (black arrow), suggesting sluggish flow but patency of the superior IJV at the skull base. More inferior portions of the IJV in the neck could not be assessed on contrast-enhanced CT, which images only the head, so it is unclear whether the remainder of the IJV was thrombosed or patent but with slow flow. No follow-up imaging was available in this patient, who had a right central venous catheter (not shown), which may have provided a nidus for thrombosis or impeded venous drainage causing slow flow.
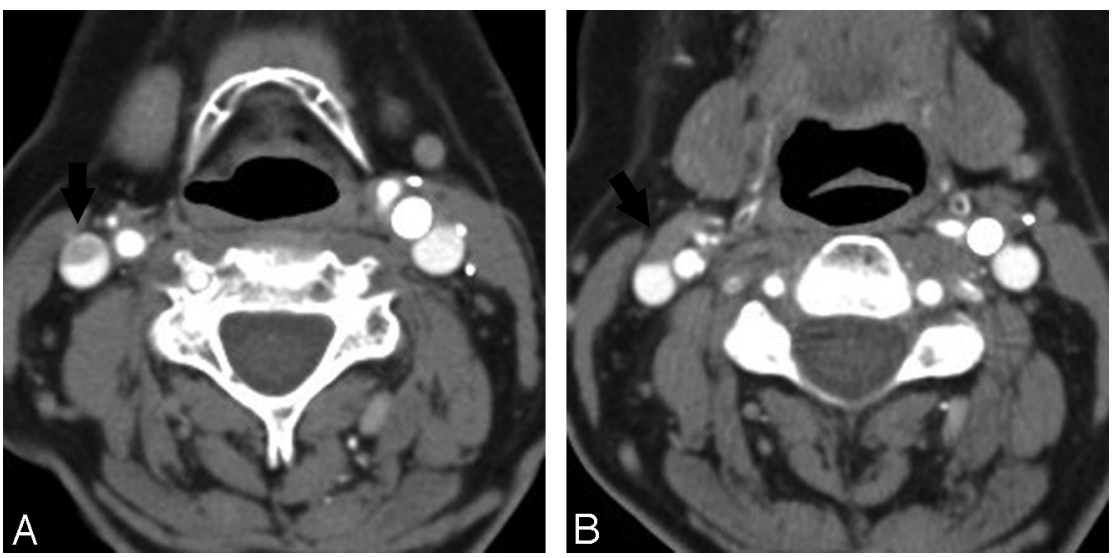

Fig 8. A 69-year-old woman with venous pseudothrombosis. A, Axial source CTA image shows an apparent eccentric filling defect in the right IJV (arrow). B, Nonopacified blood in a small vein (black arrow) draining into the IJV is responsible for causing these flow artifacts.

gree of opacification between arteries and adjacent veins could be obtained. Second, we identified important artifacts that may be specific to very fast scanning techniques such as the 64-section multidetector scanner used in this study. In particular, important artifacts related to flow dynamics produced an appearance that simulated arterial occlusion and dissection. 
These flow phenomena, while recognizable as artifacts to the radiologist experienced in interpreting 64-section CTA, could be a source of potential confusion for those not familiar with very fast scanning techniques because these artifacts have not been systematically reviewed or reported in the literature, to our knowledge. None of these artifacts were seen in our historical control group scanned on 16-section CT. Identification of these flow artifacts may be important for developing strategies to prevent these artifacts on 64-section scanners and for developing scanning protocols and interpreting images from the next generation of scanners using even higher numbers of CT detectors.

Proper timing of contrast material administration is a key determinant of optimal arterial opacification and CTA image quality. With multidetector scanners of up to 16 sections, a fixed delay (eg, 25 seconds) between contrast material injection and the start of scanning has been successfully used by some authors. ${ }^{8}$ However, given the physiologic variability of blood flow among patients (especially those with cardiac dysfunction or vascular stenoses) and the very fast imaging times of 64-section CT, accurately timing the delay between contrast material administration and scanning initiation with either a bolus-tracking technique or a preprocedure timing bolus becomes important.9-11

In bolus tracking, the main contrast material bolus is injected and then a designated vessel of interest is monitored in real-time with low-dose dynamic scanning. When a certain enhancement threshold is reached in the monitored vessel, the table moves to the start position and scanning begins. The main drawback of this technique is the inherent delay between when the threshold value is reached and when scanning begins (approximately 4 seconds), as well as the delay in computer reconstruction of bolus-tracking images (2 seconds). The combined 6-second lag often requires that an increased amount of contrast material be injected.

The timing-bolus approach circumvents this delay intrinsic to bolus tracking. In our stroke protocol, the dynamic low milliampere/kilovolt perfusion CT doubles as the timingbolus scan. Because there is no intrinsic delay with the timing-bolus approach, the volume of contrast material given for the CTA can be kept as low as possible $(70 \mathrm{~mL}$ for CTA in our protocol). With appropriate modifications to optimally time scanning delay, timing-bolus and bolus-tracking techniques both yield high-quality CTA studies.

In a study that used a phantom model of carotid stenosis and nonionic contrast material, other authors have shown that contrast densities between 150 and $200 \mathrm{HU}$ yield the most accurate assessments of stenoses on CTA. ${ }^{5}$ With our carotid CTA protocol, high arterial opacification was achieved in this study, with a mean arterial attenuation of approximately $310 \mathrm{HU}$ and attenuation values $>150 \mathrm{HU}$ in approximately $97 \%$ of arterial segments. Because ours was a segmental analysis of contrast opacification, if studies containing pseudoocclusions or pseudodissections had segments with arterial attenuation $>150 \mathrm{HU}$, these segments were included in the calculation. Of the 31 arterial segments with attenuation $<150 \mathrm{HU}$, nearly half were found in the proximal CCA, which is particularly prone to attenuation artifacts between the shoulders and artifactual lowering of contrast densities. Indeed, the proximal CCA had the lowest mean attenuation of all locations. The distal CCA and proximal ICA had the highest mean attenuation values, which are the sites of greatest clinical relevance. de Monyé et $\mathrm{al}^{12}$ had similar carotid artery attenuation values in a study of contrast material volumes and bolus chaser on 16-section CTA.

Our CTA protocol also minimized contrast opacification of cervical veins and provided high-attenuation differences between arteries and veins, with a mean attenuation difference of approximately $181 \mathrm{HU}$. Of the segments with poor image contrast between arterial and venous segments (attenuation difference $<50 \mathrm{HU}$ ), nearly half ( 23 of 49 ) of the segments were in the high cervical region, presumably because of greater time for contrast material circulation given our caudocranial imaging technique.

We generally attempted to administer intravenous contrast material from a right-sided injection for 2 reasons. First, the left brachiocephalic vein takes a more transverse course into the superior vena cava, which produces a higher likelihood of undiluted contrast material obscuring the origins of the great vessels. Second, compression of the brachiocephalic vein by an ectatic aorta, which is especially common in elderly patients, who compose a large percentage of patients undergoing CTA of the carotid arteries, can lead to contrast material pooling and subsequent reflux into neck veins. Nonetheless, comparison of right- and left-sided injections in our study did not reveal a significant difference in the extent of streak artifacts obscuring great vessel origins or median length of reflux in the IJV. de Monyé et $\mathrm{al}^{13}$ similarly did not find a significant difference in streak artifacts or length of IJV reflux between rightand left-sided injections in patients who underwent caudocranial CTA scanning.

An important finding in our study is that of flow-related artifacts that mimic arterial dissection or arterial occlusion, which were observed only on 64-section CTA and not in our 16-section CTA control group. A presumed pseudodissection flap extending vertically from the dorsal wall of the carotid bifurcation and toward the proximal ICA was observed in 3 patients. While the precise mechanism underlying this appearance is not known, we will consider several possible explanations. Several authors describe flow reversal in the carotid bifurcation at the site where pseudodissection was most commonly observed in our study. Flow reversal occurs when laminar flow in the CCA is disrupted as flowing blood enters a region of increased vessel caliber. Slower flowing blood at the periphery of the vessel lumen encounters a larger pressure gradient at the carotid bifurcation, which reverses the direction of flow. Flow reversal can appear as a linear stripe at the periphery of the bifurcation, opposite the origin of the external carotid artery, and can extend into the proximal ICA as depicted on color Doppler sonography and even angiography. ${ }^{14,15}$

A second type of flow phenomenon mimicking dissection on MR angiography is described by Saloner et al. ${ }^{16}$ The authors noted that slower flowing blood at the periphery of the lumen proximal to a $90^{\circ}$ bend becomes rotated into the center of the lumen by secondary flow and appears as a hypointense intraluminal stripe on MR angiography. Because faster imaging times are achieved with 64-section CTA, we may discern complex flow phenomena previously not captured on 4- or 16-section scanners. Alternatively, if we presume that the col- 
umn of flowing blood within the vessel is uniformly opacified, perhaps other explanations such as beam-hardening artifacts at the carotid bulb (related to calcified atherosclerotic plaque) may account for the linear low-attenuation flap that is seen.

The most common flow artifacts on CTA, pseudoocclusion and poor sensitivity to slow flow, were also the likely result of very fast scanning times because they were not seen on 16-section CTA. With 64-section CTA, areas of delayed blood flow and altered fluid dynamics (secondary to proximal stenosis, distal thrombosis, or even a central venous catheter) were rapidly imaged before adequate contrast opacification could occur. The scanning had "outrun" the contrast material bolus in areas of slow flow, which were able to opacify by the time delayed contrast-enhanced CT was performed. The emphasis of our protocol on obtaining optimal arterial enhancement on CTA images and minimizing the volume of contrast material used likely contributed to these flow artifacts. As stated earlier, we generally prefer the timing-bolus approach to bolus tracking because CTA can be performed in a pure arterial phase, with optimal enhancement of the carotid and vertebral arteries and minimal enhancement in the cervical veins. In addition, reducing the overall amount of contrast material that is administered is particularly important for patients with impaired or borderline renal function.

There were no cases of patient motion artifacts in our series, likely as a result of the very fast scanning time (3-4 seconds) afforded by 64-section CTA. While not statistically significant, 3 of the 113 studies in the 16-section CTA control group were affected by motion artifacts. The literature reports even higher rates of motion artifacts on CTA studies acquired on slower earlier generation CT scanners, including 5 of 22 cases $(22.7 \%)$ in 1 study of single-section CTA with a scanning time of 60 seconds. ${ }^{17}$

A limitation of our study is the lack of consistent follow-up imaging in patients with flow-related artifacts. Among the 6 cases of overestimation of arterial occlusion by CTA, only 3 patients had follow-up imaging to verify the assessment made on contrast-enhanced CT. Future studies might benefit from greater correlation with other noninvasive imaging techniques or with subsequent imaging in selected patients.

One consideration that merits some discussion is the radiation dose incurred by patients from our 64-section stroke protocol, which consists of pre- and postcontrast head CT, CTA, and perfusion CT. The perfusion CT is performed at a low radiation dose $(80 \mathrm{kV}, 100 \mathrm{~mA})$ and doubles as our timing bolus for CTA. The utility of perfusion CT in the acute stroke setting for predicting the extent of infarct and clinical outcome has been demonstrated in multiple studies. ${ }^{18-21}$ Finally, we also attempted to minimize the radiation dose to the patient by performing $\mathrm{x}-\mathrm{y}$-z-axis dose modulation on 64-section CT, in which the tube current applied within each CT section varies depending on the patient's attenuation and girth at each level, while still preserving image quality. ${ }^{22,23}$ With dose modulation, the radiation dose to the patient from 64-section CTA is comparable with the dose from unmodulated 16-section CTA. ${ }^{23}$ This is important given the concern for increased radiation from obtaining a higher number of very thin sections on 64-section CT scanners as compared with earlier generation scanners.

\section{Conclusions}

We have validated a CTA protocol suitable for imaging with a 64-section multidetector CT scanner and have identified 2 types of flow-related artifacts that are pronounced by using a 64-section scanner. These artifacts may be particular to very rapid CT scanning, such as that afforded by a 64 -section scanner. Recognition and understanding of these artifacts to avoid misdiagnosis should be valuable for radiologists who are performing CTA on a 64-section scanner.

\section{References}

1. Koelemay MJ, Nederkoorn PJ, Reitsma JB, et al. Systematic review of computed tomographic angiography for assessment of carotid artery disease. Stroke 2004;35:2306-12

2. Schellinger PD, Fiebach JB, Hacke W. Imaging-based decision making in thrombolytic therapy for ischemic stroke: present status. Stroke 2003;34: 575-83

3. Truwit CL. CT angiography versus MR angiography in the evaluation of acute neurovascular disease. Radiology 2007;245:362-66, discussion 366

4. Seidenwurm D, Turski P, Barr J, et al. Performance measures in neuroradiology. AJNR Am J Neuroradiol 2007;28:1435-38

5. Claves JL, Wise SW, Hopper KD, et al. Evaluation of contrast densities in the diagnosis of carotid stenosis by CT angiography. AJR Am J Roentgenol 1997; 169:569-73

6. Rubin GD, Schmidt AJ, Logan LJ, et al. Multi-detector row CT angiography of lower extremity arterial inflow and runoff: initial experience. Radiology 2001;221:146-58

7. Tan JC, Dillon WP, Liu S, et al. Systematic comparison of perfusion-CT and CT-angiography in acute stroke patients. Ann Neurol 2007;61:533-43

8. Sheikh RG, Lev MH. Stroke CT angiography (CTA). In: Gonzalez RG, Hirsch JA, Koroshetz WJ, Lev MH, et al, ed. Acute Ischemic Stroke. New York: SpringerVerlag; 2006:265

9. Bae KT. Test-bolus versus bolus-tracking techniques for CT angiographic timing. Radiology 2005;236:369-70, author reply 370

10. Cademartiri F, Nieman K, van der Lugt A, et al. Intravenous contrast material administration at 16-detector row helical CT coronary angiography: test bolus versus bolus-tracking technique. Radiology 2004;233:817-23

11. Hallett RL, Fleischmann D. Tools of the trade for CTA: MDCT scanners and contrast medium injection protocols. Tech Vasc Interv Radiol 2006;9:134-42

12. de Monyé C, Cademartiri F, de Weert TT, et al. Sixteen-detector row CT angiography of carotid arteries: comparison of different volumes of contrast material with and without a bolus chaser. Radiology 2005;237:555-62

13. de Monyé C, de Weert TT, Zaalberg W, et al. Optimization of CT angiography of the carotid artery with a 16-MDCT scanner: craniocaudal scan direction reduces contrast material-related perivenous artifacts. AJR Am J Roentgenol 2006; 186:1737-45

14. Middleton WD, Foley WD, Lawson TL. Flow reversal in the normal carotid bifurcation: color Doppler flow imaging analysis. Radiology 1988;167:207-10

15. Schuierer G, Huk WJ. Diagnostic significance of flow separation within the carotid bifurcation demonstrated by digital subtraction angiography. Stroke 1990;21:1674-79

16. Saloner D, van Tyen R, Dillon WP, et al. Central intraluminal saturation stripe on MR angiograms of curved vessels: simulation, phantom, and clinical analysis. Radiology 1996;198:733-39

17. Randoux B, Marro B, Koskas F, et al. Carotid artery stenosis: prospective comparison of CT, three-dimensional gadolinium-enhanced MR, and conventional angiography. Radiology 2001;220:179-85

18. Koenig M, Klotz E, Luka B, et al. Perfusion CT of the brain: diagnostic approach for early detection of ischemic stroke. Radiology 1998;209:85-93

19. Lev MH, Segal AZ, Farkas J, et al. Utility of perfusion-weighted CT imaging in acute middle cerebral artery stroke treated with intra-arterial thrombolysis: prediction of final infarct volume and clinical outcome. Stroke 2001;32: 2021-28

20. Mayer TE, Hamann GF, Baranczyk J, et al. Dynamic CT perfusion imaging of acute stroke. AJNR Am J Neuroradiol 2000;21:1441-49

21. Ezzeddine MA, Lev MH, McDonald CT, et al. CT angiography with whole brain perfused blood volume imaging: added clinical value in the assessment of acute stroke. Stroke 2002;33:959-66

22. Smith AB, Dillon WP, Gould R, et al. Radiation dose-reduction strategies for neuroradiology CT protocols. AJNR Am J Neuroradiol 2007;28:1628-32

23. Smith AB, Dillon WP, Lau BC, et al. Radiation dose reduction strategy for CT protocols: successful implementation in neuroradiology section. Radiology 2008;247:499-506 